\title{
Toxic epidermal necrolysis: Revisiting the tentative link between early apoptosis and late necrosis (Review)
}

PHILIPPE PAQUET and GERALD E. PIERARD

\author{
Department of Dermatopathology, University Hospital of Liège, Liège, Belgium
}

Received June 23, 2006; Accepted August 30, 2006

\begin{abstract}
Toxic epidermal necrolysis (TEN) is a dramatic drug-induced reaction that may lead to full destruction of the epidermis and epithelial mucosae. The keratinocytes themselves seem to play a major role in the pathogenic mechanism. Biochemical and morphological studies performed on early epidermal lesions demonstrated that keratinocytes undergo apoptosis, but histological and clinical data show evidence of necrosis of the epidermis later in the disease evolution. Based on the limited information currently available about TEN pathomechanism, we present a 'mitochondrial hypothesis' that may explain both early apoptosis and late necrosis in TEN epidermis. Strong electrophilic drug metabolites are generated in TEN-affected keratinocytes due to an impaired detoxication pathway. These compounds presumably disrupt the electron transfer chain in the mitochondriae resulting in a decline in ATP production, loss of electrochemical gradient across the inner membrane $(\Delta \psi \mathrm{m})$, and partial reduction in $\mathrm{O}_{2}$ with production of reactive oxygen species (ROS). The latter compounds directly damage cell membranes and act as intracellular chemical messengers stimulating proapoptotic systems such as CD95 and TNF- $\alpha$, which in turn can activate nitric oxide (NO) metabolism. NO interacts with ROS to enhance their toxic effects. These proapoptotic events represent swift processes in the involved cells. The loss of $\Delta \psi \mathrm{m}$ and the opening of permeability transition pores in the mitochondrial membrane lead to osmotic swelling and rupture of these organelles with subsequent necrosis of the cell. The necrotic events follow apoptosis when both phenomena are present.
\end{abstract}

\section{Contents}

1. Introduction

2. Key role of keratinocytes in TEN

Correspondence to: Dr Philippe Paquet, Department of Dermatopathology, CHU Sart Tilman, B-4000 Liège, Belgium

E-mail: p.paquet@chu.ulg.ac.be

Key words: necrosis, apoptosis, toxic epidermal necrolysis
3. Apoptosis

4. Necrosis

5. Tentative physiopathological pathway of TEN epidermal destruction

6. Conclusion and future prospects

\section{Introduction}

Drug-induced toxic epidermal necrolysis (TEN), also known under its eponym Lyell's syndrome, currently remains one of the most dramatic dermatological emergencies. This lifethreatening disease is characterized by extensive destruction of the epidermis and mucosal membranes. The yearly incidence of TEN is estimated at approximately $2-3$ cases per $10^{6}$ individuals. The $25-30 \%$ TEN mortality rate is mainly due to secondary septicemia and metabolic failures following loss of epidermal integrity (1).

A large body of evidence suggests that TEN is an adverse drug reaction resulting from a specific alteration of drug metabolism. More than 100 different drugs may cause TEN, but only a minority accounts for the vast majority of cases. These are mainly represented by antibacterial sulfonamides (co-trimoxazole), aromatic anticonvulsants (phenobarbital, phenytoin and carbamazepine), non-steroidal anti-inflammatory agents (phenylbutazone, oxyphenbutazone, isoxicam and piroxicam), allopurinol and various antibiotics (2).

Biochemical and morphological features of apoptosis have been demonstrated in TEN-involved keratinocytes during the initial stage of the disease. This was shown by electron microscopy, by the characteristic ladder pattern of DNA cleavage, and by the presence of terminal deoxynucleotidyl transferase-mediated DNA nick end labelling in keratinocytes (3). However, the final morphological characteristic of apoptosis, i.e. the formation of apoptotic bodies, is not found in TEN epidermis. By contrast, the late phase of TEN is characterized by full-thickness epidermal necrosis. Hence, it is assumed that the TEN pathomechanism likely combines early apoptosis and late necrosis. The involvement of mitochondriae in TEN keratinocytes may explain the simultaneous presence of these two death mechanisms in TEN epidermis. For a better understanding of this 'mitochondrial hypothesis', we review the key role of keratinocytes in TEN pathobiology, and we tentatively integrate the main mechanisms of apoptosis and necrosis. 


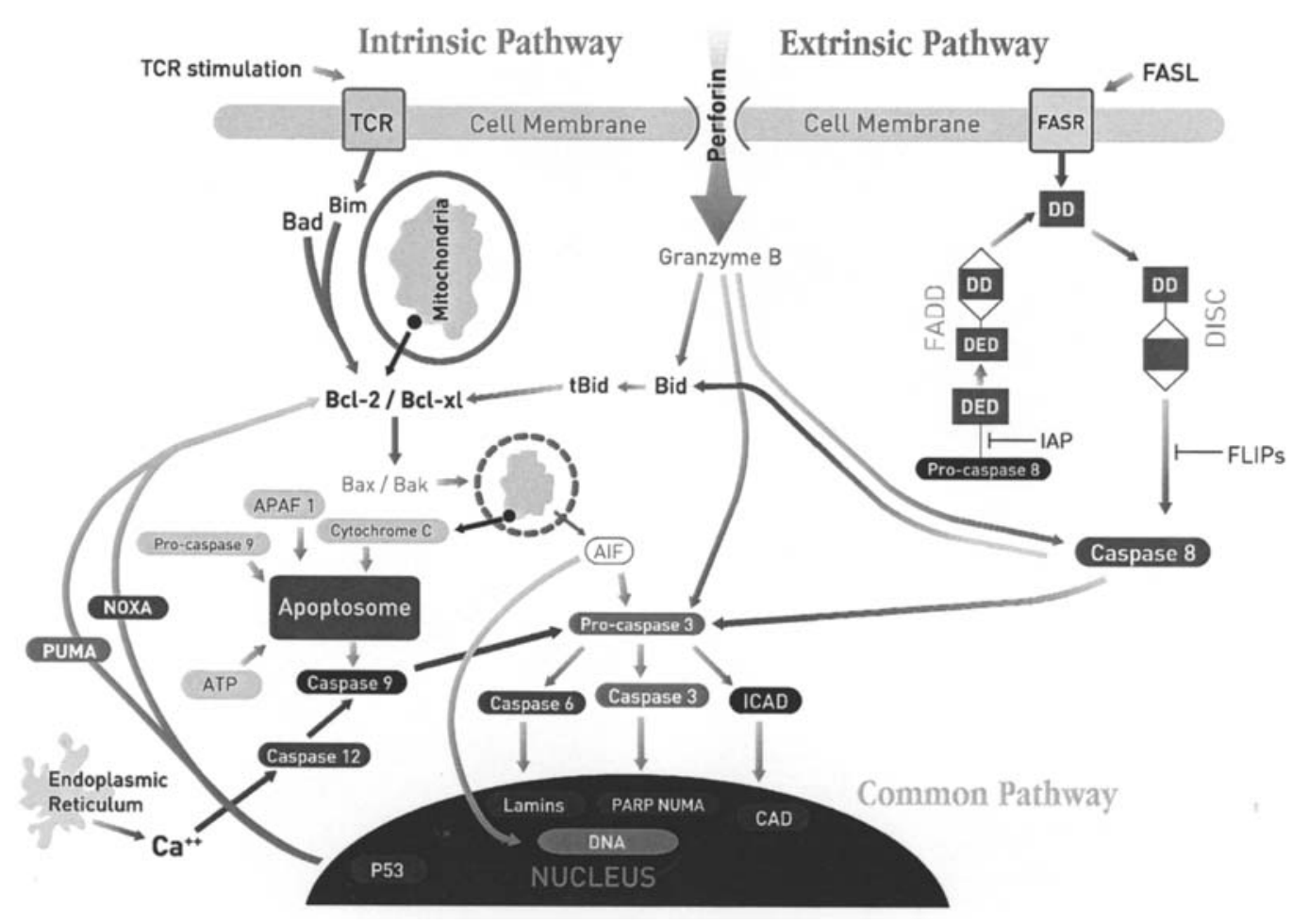

Figure 1. Apoptosis pathomechanisms.

\section{Key role of keratinocytes in TEN}

Although activated $\mathrm{T}$ lymphocytes and macrophages undoubtedly participate in the TEN epidermal destruction, they may well only represent ancillary effectors. Indeed, keratinocytes seem to represent the key initial protagonists in TEN epidermal destruction. A series of arguments supports this pathogenic hypothesis. First, keratinocytes are increasingly recognized for their metabolic activity through various transport-associated and detoxifying enzymes (4). The constitutive presence of the cytochrome fraction CP450 3A and the potential induction of CP450 1A are well documented in the human epidermis. As an example, covalent binding of carbamazepine-reactive metabolites to CP450 isoforms was demonstrated in TEN epidermis (5). Second, most TEN lesions show a minimal inflammatory cell infiltrate (6). Moreover, there is overexpression of different proapoptotic systems by TEN keratinocytes, including tumor necrosis factor $\alpha(\mathrm{TNF} \alpha)(7)$, and Fas receptor/Fas ligand (CD95R/ CD95L) (8). Functional studies in vitro have demonstrated that keratinocytes from TEN patients express lytically active Fas-L (8). The cytosolic complex calprotectin (L1-protein) is also almost always expressed both in clinically involved and apparently uninvolved TEN epidermis (9). The L1-protein results from a calcium $\left(\mathrm{Ca}^{++}\right)$-related process. Its presence in normal-looking epidermis of TEN patients in the absence of inflammatory cells suggests that an increase in $\mathrm{Ca}^{++}$ concentration inside the keratinocytes is one of the earliest biological events disclosed in the process of TEN destruction following the drug metabolite(s) formation.

To note, a unique case of antibiotic-induced TEN was reported in a patient who also suffered from prolonged severe methotrexate-induced pancytopenia without any circulating leucocytes (10). In this case, T lymphocytes and macrophages were absent in the epidermis. This observation suggests that the keratinocytes were responsible for their own destruction without the intervention of inflammatory cells.

\section{Apoptosis}

Apoptosis represents one major mechanism physiologically involved in tissue involution and in reducing the expansile growth of cancers $(11,12)$. This process can be regarded as a programmed cell death which is expressed by a distinct set of biochemical, morphological and immunohistochemical alterations. Apoptosis is characterized by DNA fragmentation into oligonucleosome-sized fragments. It is recognized morphologically by cell shrinkage, chromatin condensation along the periphery of the nucleus, and the formation of apoptotic bodies. This entire process keeps well preserved the internal and external cell membranes. In most instances, apoptotic bodies are rapidly phagocytosed by adjacent cells and macrophages.

These specific changes contrast with necrosis following severe lethal insult. In this process, cells undergo osmotic swelling and lysis with loss of membrane integrity. The chromatin tends not to condense at the nucleus periphery, but it clumps within the nucleus or disperses rapidly with dissolution of the nuclear membrane. Thus, the cellular content is released into the interstitium during necrotic cell death.

Apoptosis is induced by a series of physiological factors including some cytokines, and by some chemotherapy regimens and radiation therapy. Apoptosis involves the activation of a complex array of related aspartate-specific cysteine-proteases corresponding to caspases, either through death receptors or mitochondria-mediated apoptotic events (Fig. 1). 
Mitochondria-mediated apoptosis (intrinsic pathway). A variety of cellular insults like TCR stimulation or cytokine deprivation promote the activation or the expression of proapoptotic Bcl-2 family members including Bid (Bcl-2 interacting domain), Bad (Bcl-2-associated death promotor), Bim (Bcl-2 interacting mediator of cell death), PUMA (p53 upregulated modulator of apoptosis) and NOXA (after the latin word for damages) $(11,12)$.

Once induced, these proteins inhibit the antiapoptotic Bcl-2/Bcl-XL complex, allowing the oligomerization of the proapoptotic system Bax and Bak (Bcl-2-associated X protein and Bcl-2 antagonist killer-1). The freed Bax/Bak system creates pores in the mitochondrial outer membrane, thus altering the mitochondrial permeability. The leakage of constituents like cytochrome $\mathrm{C}$ (Cyt C) ensues in the cytosol. This process promotes the assembly of the apoptosome corresponding to a multi-protein complex combining Cyt C, its adapter Apaf-1 (apoptotic protease activating factor 1), procaspase 9 and ATP necessary for the oligomerization of Apaf 1. Procaspase 9 is autoactivated and cleaves downstream executioner procaspase 3 . Other released mitochondrial constituents involved in apoptosis include SMAC/DIABLO (second mitochondria-derived activator of caspase/direct IAP binding protein with low PI), OMI (homologue of bacterial $\mathrm{Htr} \mathrm{A}$ ) and endonuclease $\mathrm{G}$.

Another caspase-activating protein released from mitochondriae is AIF (apoptosis inducing factor) which processes procaspase 3 (Fig. 1). The AIF release can also lead to chromatin condensation irrespective of caspase activation. The kinetics of mitochondrial constituent breakage may determine whether caspase-dependent or caspaseindependent pathways are recruited. In the presence of Cyt C translocation, the caspase cascade may dominate by value of the rapid rate of activation, whereas in its absence or with inhibition of the caspase cascade, non-caspase-dependent AIF activity may proceed to cell death.

Death receptor-mediated apoptosis (extrinsic pathway). Death receptor-mediated apoptosis is induced by the activation of specific molecules including TNF receptors (TNF-R), TNF-related apoptosis-inducing ligand receptor (TRAIL-R) and fibrin adhesive system receptors (FasR or CD95R) (Fig. 1). Ligation of the receptors transmits the apoptotic signals through structures called death domains (DD) and death effector domains (DEDs) $(11,12)$.

DD are found in transmembrane proteins, including FasR, TNF-R1, TRAIL-R1/DR4, and TRAIL-R2/DR5. They are also present in cytoplasmic proteins including the bipartite activator molecule Fas-associated protein with death domain (FADD), TNF receptor-associated protein with death domain (TRADD), and receptor-interacting protein (RIP).

DEDs are found in the adaptor molecules as well as in procaspases 8 and 10. They play a prominent role in death receptor-induced apoptosis where the interactions between the DED of the adaptor molecules and of procaspases 8 or 10 produce the death-inducing signalling complex (DISC) representing a complex of apoptosis-inducing proteins. The binding of DISC to procaspase 8 initiates the caspase cascade.

These different pathways are regulated by FLIPs [FADDlike ICE (FLICE) inhibitory proteins] and IAPs (inhibitor of

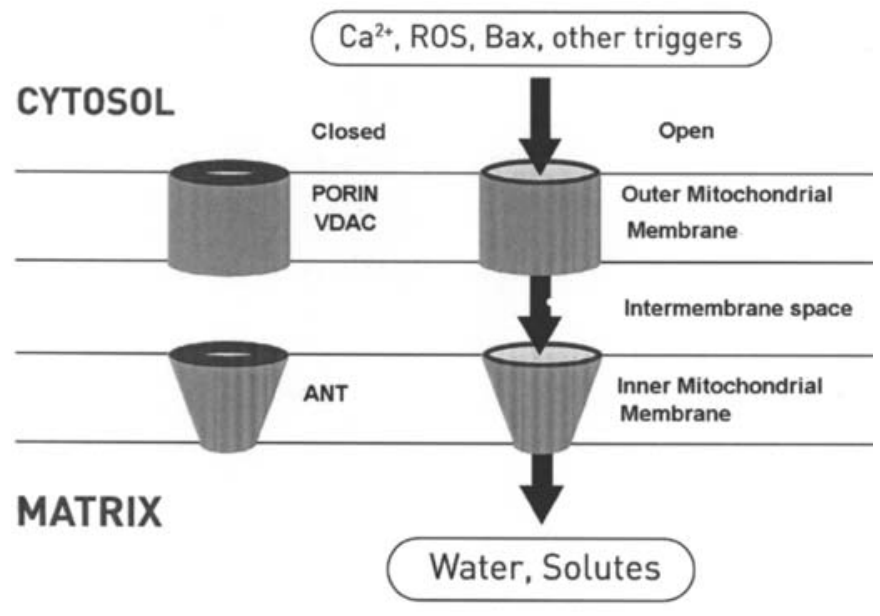

Figure 2. Structure of the mitochondrial PT pore.

apoptosis proteins) such as survivin. FLIPs resemble procaspase 8 in containing the DEDs, but lack the catalytic domain. They can therefore inhibit death-receptor signalling by usurping procaspase 8 sites on the DISC. IAPs bind to inactive caspases.

Additional pathways of apoptosis. Apoptosis can be triggered by stressors to the endoplasmic reticulum that disrupt $\mathrm{Ca}^{++}$ homeostasis and involve caspase 12 , thus coupling the dysfunction of the endoplasmic reticulum to mitochondriamediated apoptosis (Fig. 1) $(13,14)$. Caspase 12 can also directly cleave and activate procaspase 9 , bypassing $(13,14)$ the requirement for $\mathrm{Cyt} C$ release. In addition, released $\mathrm{Ca}^{++}$ activates calpains, which in turn cleave and activate Bax directly (Fig. 1).

Link between the intrinsic and extrinsic pathways. The integration between extrinsic and intrinsic pathways of apoptosis operates through Bid (Fig. 1). The active caspase 8 can cleave Bid into the truncated active form tBid. The latter displaces Bax from inactive heterodimers with Bcl-2 or $\mathrm{Bcl}-\mathrm{XL}$, allowing the apoptosis to proceed $(11,12)$.

Moreover, the granular components of cytotoxic $\mathrm{T}$ cells and NK cells contain perforin mediating pore formation in the target cell membrane. This process facilitates the entry of the serine protease granzyme B which can target caspases 3 and 8 , and Bid.

Final common pathway of apoptosis. Extrinsic and intrinsic caspase-dependent pathways of apoptosis lead to the activation of procaspase 3 (Fig. 1). This mechanism represents the final common pathway leading to apoptosis directly or through caspase 6 activation. Key substrates of caspase 3 include the inhibitor of caspase-activated DNase (ICAD), poly(ADPribose) polymerase (PARP) and the lamins. ICAD normally sequesters the DNase, CAD/DNA fragmentation factor-40 (DFF-40) in an inactivated status, until caspase 3 cleaves ICAD, allowing CAD to carry out internucleosomal degradation of the genomic DNA. PARP is involved in DNA repair $(11,12)$. 


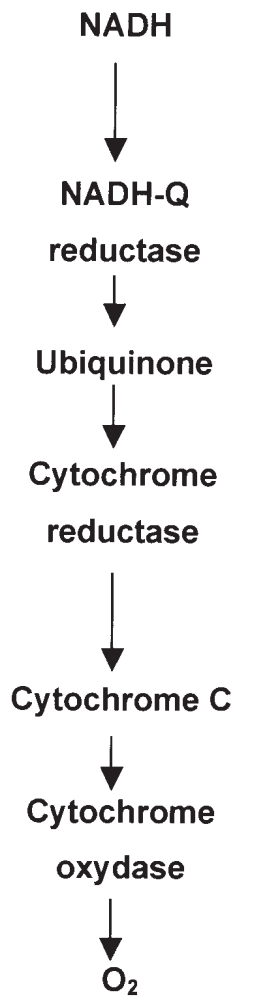

Figure 3. Sequence of the electron carriers in the respiratory chain.

Nuclear structural proteins including lamins that constitute the nuclear lamina, and nuclear mitotic apparatus protein 1 (Numa) which helps to organize the mitotic spindle, are also targets for caspase 3 and caspase 6 activity. Together, these molecules appear to coordinate and execute the nuclear changes observed during apoptosis.

\section{Necrosis}

Apart from severe direct lethal insult, a slower process resembling necrosis can be initiated by the mitochondriae $(15,16)$. Indeed, these organelles possess large conductance channels called permeability transition (PT) pores (Fig. 2). The structure of these pores includes both inner membrane proteins such as the adenine nucleotide translocator (ANT) normally involved in ATP and ADP exchange across the mitochondrial inner membrane, and outer membrane proteins such as porin (voltage-dependent anion channel, VDAC). These inner and outer membrane proteins operate in concert and create a channel through which molecules $\leq 1.5 \mathrm{kDa}$ can pass through. Opening the ANT channels in the inner membrane allows for an equilibration of ions within the matrix and intermembrane space of mitochondriae, thus dissipating the $\mathrm{H}^{+}$gradient across the inner membrane $(\Delta \psi \mathrm{m})$ with subsequent deregulation in the electron transport in the respiratory chain (Fig. 3), ATP depletion (Fig. 4) and the shunting of electrons into the generation of ROS, such as $\mathrm{O}_{2}^{-0}$. PT pore opening also results in a deregulation of the mitochondrial size due to hyperosmolarity of the matrix which causes the matrix space to expand and to rupture the outer membrane, releasing caspase-activating protein located within the intermembrane space into the cytosol.

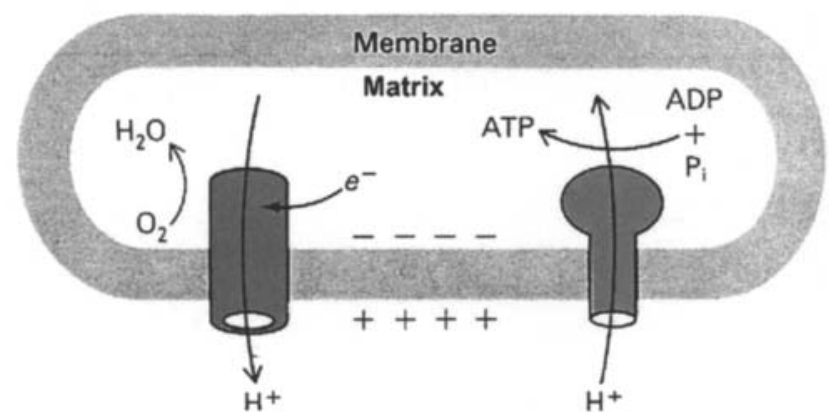

Figure 4. Oxidative phosphorylation in the mitochondria. Oxidation and ATP synthesis are coupled by a transmembrane flux of protons.

Bcl-2 prevents the ANT opening whereas Bax induces it. Other stimuli that affect the PT pore directly such as oxidant and pathological elevation in cytosolic $\mathrm{Ca}^{++}$can induce the rupture of the outer membrane of mitochondriae and the release of Cyt $\mathrm{C}$. Pharmacological agents inhibiting the channel opening attenuate apoptosis and/or necrosis, while drugs triggering ANT opening may induce these mechanisms $(15,16)$.

\section{Tentative physiopathological pathway of TEN epidermal destruction}

Formation of electrophilic toxic compounds. Severe adverse drug reactions have been especially studied pharmacologically with two important types of drugs, namely the sulfonamide antibiotics (co-trimoxazole) and the aromatic anticonvulsants (phenytoine, carbamazepine and phenobarbital).

The pathway of sulfonamide metabolism has been primarily studied in vitro using a lymphocyte assay evaluating cell death induced by metabolites generated by a murine hepatic microsomal system (17). In this model, the sulfonamide component of co-trimoxazole, sulfamethoxazole (SMX) undergoes CP450-mediated oxidation to a toxic hydroxylamine metabolite (SMX-NOH) (Fig. 5). Further spontaneous non-enzymatic oxidation yields nitroso-SMX. The nitroso-derivative is more electrophilic, and more toxic than the hydroxylamine as it has a greater degree of covalent association with cellular macromolecules, but it is rapidly reduced to SMX-NOH by reduced glutathione (GSH) and other reducing agents (18).

Sulfonamides may also be acetylated in non-toxic metabolites by the enzymes $\mathrm{N}$-acetyltransferases (NAT), coded by two genes named NAT-1 and NAT-2 (19). The acetylation of SMX is mediated primarily either by NAT-1 or NAT-2 which are respectively the predominant NAT expressed in peripheral blood mononuclear cells and liver $(18,20)$. Finally, the balance between activation and detoxication processes may be critical for the immunogenic potential of compounds.

In TEN, the attack of the liver is generally absent or moderate with regard to the major involvement of the epidermis. Hence, it is unlikely that reactive metabolites are formed in the liver, and the bioactivation probably occurs in the keratinocytes themselves. Indeed, human keratinocytes appear capable of bioactivating SMX to reactive hydro- 


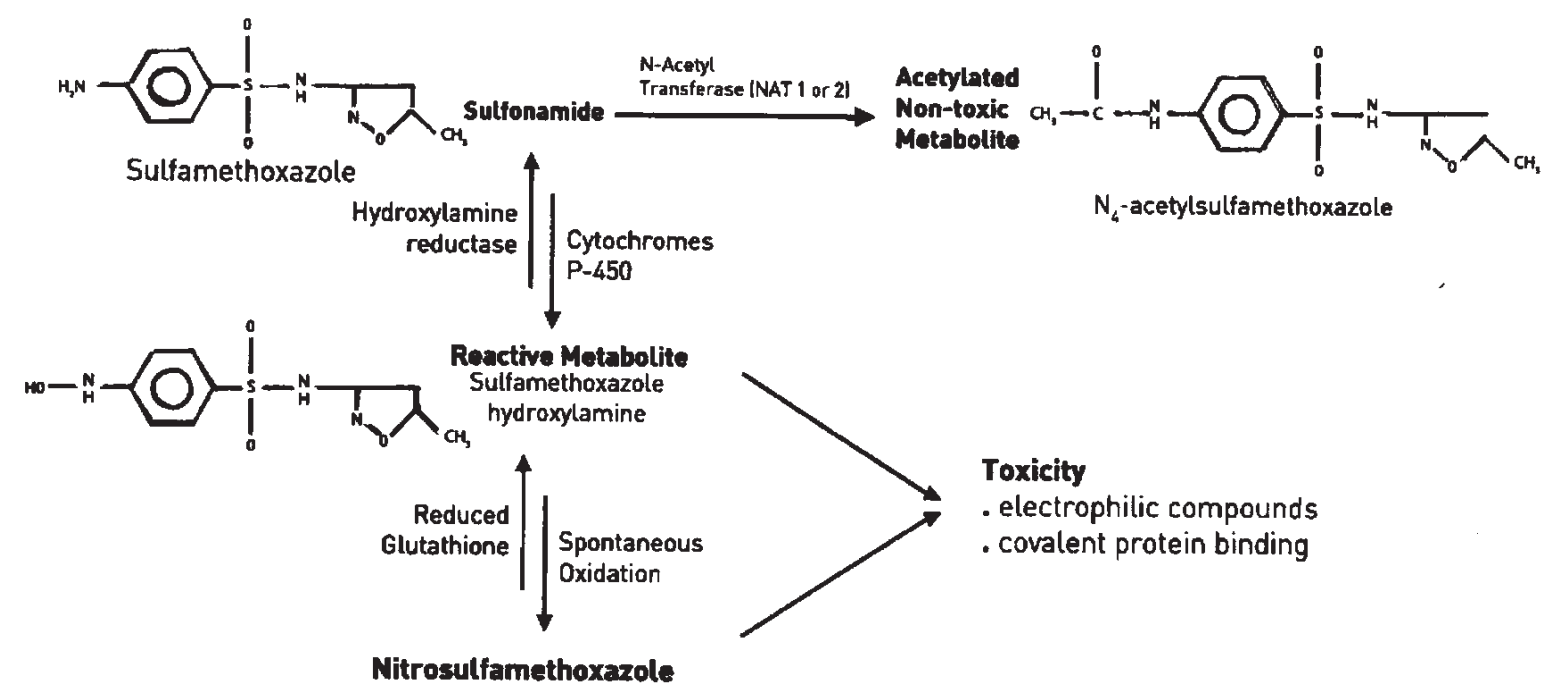

Figure 5. Schematic representation of sulfamethoxazole metabolism in peripheral blood lymphocytes and hepatocytes.

xylamine species although the enzymes mediating this process are unknown (21). Keratinocytes also possess NAT activity, apparently through NAT-1 alone (21). A decrease in cutaneous $\mathrm{N}$-acetylation activity has been reported in TEN patients (22) which may decrease cutaneous clearance of the parent drug SMX, allowing more drug to be metabolized via oxidative pathways.

In fact, human keratinocytes are resistant to the toxicity of SMX-NOH unless GSH is depleted, whereupon these cells become susceptible to its cytotoxic effects (21). GSH is important for the prevention of SMX-NOH-induced keratinocyte toxicity probably through its ability to reduce nitrosoSMX to SMX-OH and the parent drug. This may be of particular importance in HIV-infected patients who have low systemic and intracellular GSH levels $(23,24)$, and an increased risk of TEN (25).

The covalent interaction of reactive SMX-OH with cellular macromolecules has been detected in human keratinocytes and is probably a critical step in the manifestation of adverse effects (21). Major protein targets of SMX-OH are unknown but they are observed in the region of $160,125,95$ and $57 \mathrm{kDa}$ (21). These adducts can create toxic compounds or neoantigens that can be recognized by the immune system. Their production is increased with GSH depletion. If these adducts are found at low levels within viable keratinocytes, they may accumulate to high levels over the usual 7-14 days under drug therapy that commonly precede a cutaneous toxic reaction and then participate to cell demise (21). The threshold of toxicity of these adducts is expected to vary among individuals, possibly as a function of detoxication ability and may be the primary determinant for developing NET. The adducts may also form an endogenous antigen that is presented on MHC class I for recognition by $\mathrm{CD}^{+}$cells (26).

The aromatic anticonvulsant agents are metabolized to hydroxylated aromatic compounds, the arene oxides, by CP450 oxidative metabolism $(27,28)$. Arene oxides are capable of covalently binding to cellular macromolecules, thereby disturbing cell function and initiating secondary immunological responses. Arene oxides rearrange to electrophilic quinone and quinone-imine metabolites, which are also protein reactive.

Early apoptosis (Fig. 6). The generation of strong electrophilic metabolites in TEN keratinocytes may lead to the disruption of the electron transfer chain in the mitochondriae with a decline in ATP production. There is also loss of the $\Delta \psi \mathrm{m}$, and partial reduction of $\mathrm{O}_{2}$ with production of ROS. NO metabolism is altered as well.

Decrease in ATP production. Disrupting the electron transport chain alters the oxidative phosphorylation and abates the ATP production (Fig. 4). Such a metabolic drop has been observed during the late phase of apoptosis.

The production of GSH, which is a major detoxifying pathway of drug reactive metabolites in adverse cutaneous reaction, is an ATP-dependent process. Hence, any decrease in ATP production leads to impaired detoxification.

Loss of the mitochondrial inner transmembrane potential $(\Delta \psi m)$. The loss of $\Delta \psi \mathrm{m}$ envisions the opening of the large conductance channels in the outer membrane, thus releasing Cyt $\mathrm{C}$ from the intermembrane space of mitochondriae into the cytosol. Cyt $\mathrm{C}$ activates caspases following binding to Apaf-1, inducing it to associate with procaspase 9, thereby triggering caspase 9 activation and initiating the proteolytic cascade that culminates in apoptosis (Fig. 1).

Increased production of ROS. During normal transfer of electrons to $\mathrm{O}_{2}$ (Fig. 3), an estimated 1-5\% of electrons in the respiratory chain are derived to the formation of the superoxide anion $\mathrm{O}_{2}^{-\mathrm{o}}$ production $\left(\mathrm{O}_{2}+\mathrm{e}^{-} \Leftrightarrow \mathrm{O}_{2}^{-\mathrm{o}}\right)$. Any event decreasing the coupling efficiency of electron chain transport can increase the production of superoxides. However, the generation of ROS during apoptosis may represent a relatively late event, occurring after the cells are engaged in the process of caspase activation (15).

ROS act as intracellular chemical second messengers, and increase gene transcription of inflammatory cytokines via transient activation by phosphorylation or allosteric modifications of redox-sensitive nuclear transcription factors 


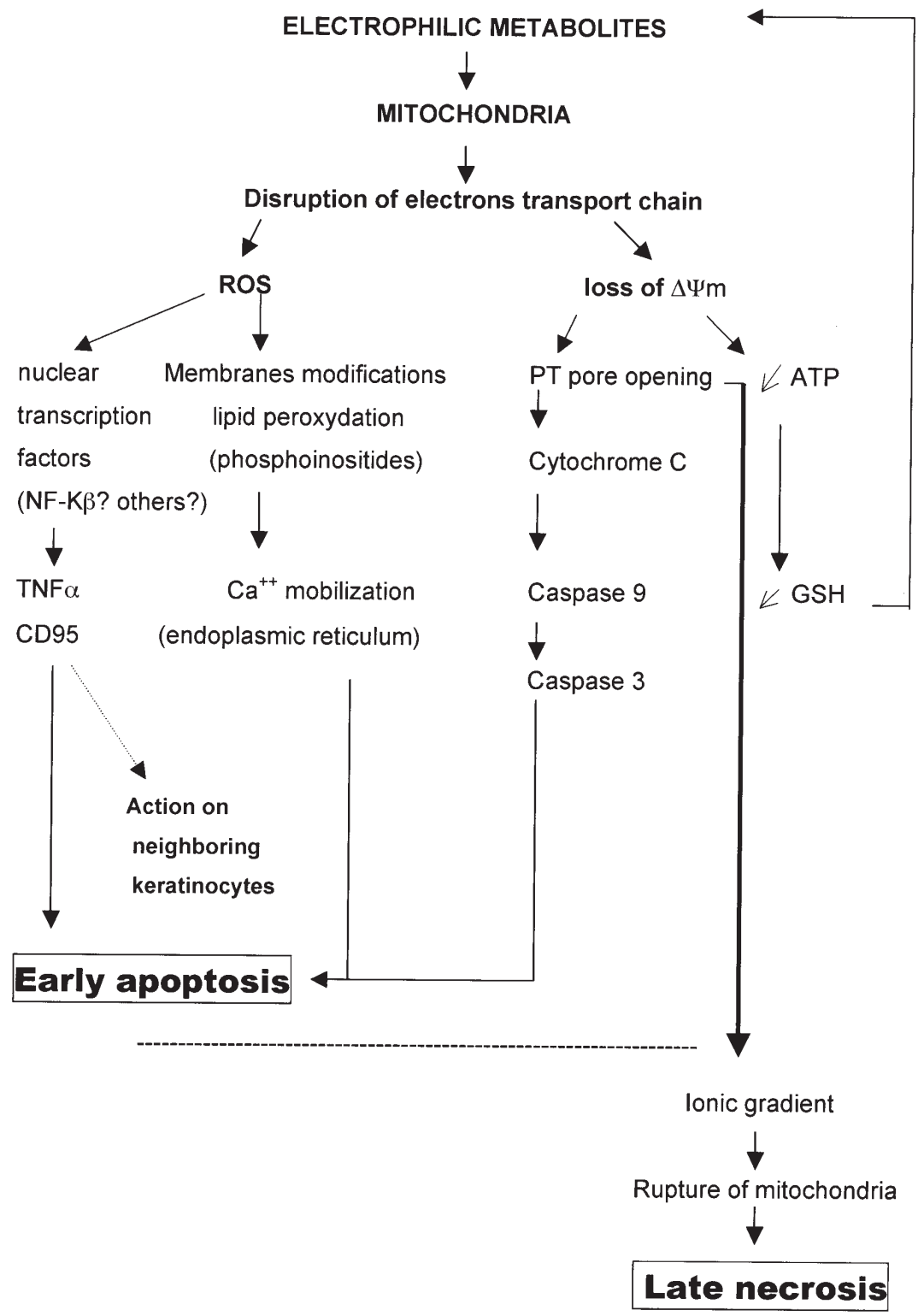

Figure 6. The TEN tentative physiopathological pathway in TEN keratinocytes.

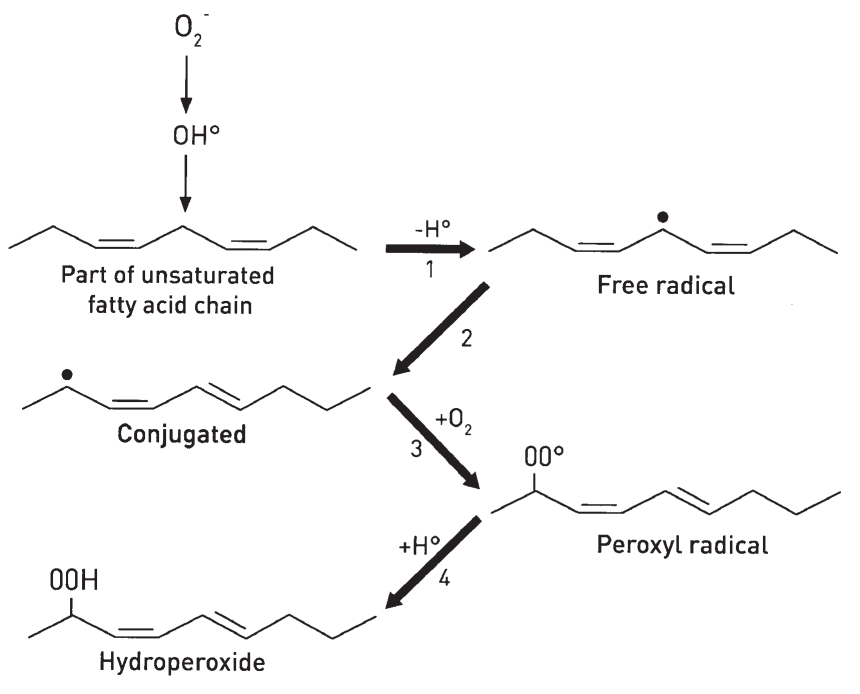

Figure 7. Peroxidation of a fatty acid chain. The first step consists of the removal of a hydrogen atom from a fatty acid chain by $\mathrm{OH}^{\circ}$. such as NF-кß, AP-1 or USF43 (29-31). In keratinocytes, ROS induce IL-6, IL-8 and TNF- $\alpha$ production (32). Indeed, a high expression of these cytokines has been found early in the course of TEN in keratinocytes and in blister fluid $(7,33,34)$. The production of these proinflammatory cytokines, especially TNF- $\alpha$ could act as an autocrine or paracrine factor to spread TEN epidermal destruction. TNF- $\alpha$ also stimulates inducible NO synthase (iNOS) leading to the production of a large amount of NO (35). Increased expression of iNOS has been demonstrated in TEN (36). Generation of ROS may also increase CD95R expression on keratinocytes (37). The involvement of the proapoptotic CD95L/CD95R system has been previously shown in TEN epidermis $(8,38)$. Moreover, complex interactions probably exist between TNF- $\alpha$ and the CD95 system (39).

In addition, ROS directly damage cellular components. The superoxide anion can accumulate in the hydrophobic portion of the cell membrane and can destroy the fatty acid esters of phospholipids, thus altering membrane semipermeability. 
The toxicity of $\mathrm{O}_{2}^{-0}$ could also pertain to its conversion into the highly reactive hydroxyl $\left(\mathrm{OH}^{\circ}\right)$ radical $(40)$. An important role for $\mathrm{O}_{2}^{-0}$ in this mechanism may be the provision of iron required for $\mathrm{OH}^{\circ}$ generation. $\mathrm{O}_{2}^{-\mathrm{o}}$ can reductively mobilize iron ions from the iron storage protein ferritine or iron-sulfur proteins. The superoxide-derived $\mathrm{OH}^{\circ}$ radical initiates lipid peroxidation by abstracting hydrogen from a fatty acid chain (Fig. 7). The latter quickly forms a carboncentered radical in combination with $\mathrm{O}_{2}$ (peroxyl radical). This can attack adjacent fatty acid side-chains and propagate lipid peroxidation. The chain reaction continues and lipid peroxides accumulate in the membranes, destabilizing them and making them 'leaky' to ions. By altering membrane phosphoinositides, ROS-induced lipid peroxidation initiates apoptotic signal cascades via the mobilization of $\mathrm{Ca}^{++}$from the endoplasmic reticulum and the activation of protein kinase $\mathrm{C}$ (32). An increase in $\mathrm{Ca}^{++}$cytosol has been shown to be an early event in TEN keratinocytes (9).

Production of $i N O S$. $\mathrm{NO}^{\circ}$ is a small diffusible molecule produced in most human tissues, especially keratinocytes (41). It is derived from the oxidation of L-arginine catalyzed by constitutive NOS or iNOS. Large amounts of iNOS are produced after challenge with lipopolysaccharides and cytokines, e.g. interferon, interleukin-1 and TNF- $\alpha$ (35). In TEN, iNOS is induced in keratinocytes, but the steps between exposure to a drug and the activation of iNOS remain unclear (36).

NO inhibits the mitochondrial respiratory chain at the level of Cyt $\mathrm{C}$ oxidase complex enhancing intracellular $\mathrm{O}_{2}^{-\mathrm{o}}$ production (42). $\mathrm{NO}^{\circ}$ may react with $\mathrm{O}_{2}^{-0}$ to generate a high level of the strong oxidant peroxinitrite anion $\mathrm{OONO}^{-}\left(\mathrm{NO}^{\circ}+\right.$ $\left.\mathrm{O}_{2}^{-\mathrm{o}} \rightarrow \mathrm{ONOO}^{-}\right)$. The latter compound may destroy Fe-S clusters of NADH-dehydrogenase and Cyt reductase complexes. It may induce lipid peroxidation thus causing irreversible damage to mitochondriae and the release of $\mathrm{Ca}^{2+}$ into the cytosol (42). Peroxinitrite anions inhibit via nitration the mitochondrial antioxidant enzyme superoxide dismutase catalyzing the reaction of ion superoxide neutralization (42).

Moreover, the $\mathrm{SH}$ group is a prime target for the covalent binding of $\mathrm{NO}^{\circ}$ to functional groups of protein in the presence of oxygen (42). A cellular thiol such as GSH has been found to react with intracellular $\mathrm{NO}^{\circ}$ yielding inactive 5-nitrosoglutathione. GSH, which is probably the most important cellular antioxidant, directly scavenges various free radicals, and serves as a substrate for the glutathione peroxidase enzyme. GSH depletion dramatically increases cellular sensitivity toward $\mathrm{NO}^{\circ}$, suggesting that intracellular GSH scavenges $\mathrm{NO}^{\circ}$ derived species (42).

Finally, $\mathrm{NO}^{\circ}$ can elicit either cellular necrosis or apoptosis. Necrosis predominates in response to acute exposure to a high $\mathrm{NO}^{\circ}$ concentration when $\mathrm{NO}^{\circ}$ can react with $\mathrm{O}_{2}^{-\mathrm{o}}$ to generate a high level of peroxinitrite anion. In contrast, prolonged exposure to low doses of $\mathrm{NO}^{\circ}$ induces apoptosis (43).

Late necrosis (Fig. 6). The loss of $\Delta \psi \mathrm{m}$ leading to PT pore opening also results in the osmotic disturbance of mitochondriae due to the hyperosmolarity of the matrix, with expansion of the matrix space, organelle swelling and subsequent rupture of the outer membrane (15).
Cells in which mitochondriae have ruptured are at risk of death through a slow nonapoptotic mechanism resembling necrosis. Apoptotic events appear earlier than necrosis when the two phenomena are present (15). TEN epidermal destruction combines apoptosis in the beginning of the disease and necrosis as a late event.

\section{Conclusion and future prospects}

We frame the hypothesis that the disruption of the electron transfer chain in mitochondriae by electrophilic metabolites of the culprit drug may be the major event leading to both early apoptosis and late necrosis in TEN. Hence, the restoration of the electron transport chain may be a key target in the treatment of TEN.

At present, no specific treatment has proven to be highly effective in TEN and to be significantly superior to the supportive care alone that remains so far the gold standard of treatment. The early withdrawal of the culprit drug decreases the amount of electrophilic metabolites. This feature is of paramount importance. It has been clinically demonstrated that the prompt withdrawal of the culprit drug is associated with a better prognosis in TEN (44). In addition, some chemical agents like $\mathrm{N}$-acetylcysteine may be useful in the treatment of TEN because they react with oxidative compounds and replenish intracellular cysteine levels necessary for the production of GSH $(45,46)$. Conversely, treatments that only block the activities of the proinflammatory cytokines and receptors produced in TEN, without acting on the deregulation of the electron transport chain in mitochondriae will probably have a limited effect on the course of TEN. This may be the case for intravenous immunoglobulins, systemic corticosteroids, cyclosporine and antiTNF- $\alpha$ agents (47). Until effective chemical treatments are developed that are able to neutralize the reactive electrophilic metabolites and to restore the electron transfer chain in TEN keratinocytes, we suggest that several blocking agents (e.g. acetylcysteine combined with intravenous immunoglobulins or anti-TNF- $\alpha$ agents) should be used simultaneously to reach optimum efficacy in the treatment of TEN.

\section{Acknowledgements}

This work was supported by a grant from the 'Fonds d'Investissement de la Recherche Scientifique' of the University Hospital of Liège. No other sources of funding were used to assist in the preparation of this manuscript. The authors have no conflicts of interest that are directly relevant to the content of this review.

\section{References}

1. Fritsch PO and Sidoroff A: Drug-induced Stevens-Johnson syndrome/toxic epidermal necrolysis. Am J Clin Dermatol 1: 349-360, 2000.

2. Roujeau JC, Kelly JP, Naldi L, et al: Medication use and the risk of Stevens-Johnson syndrome or toxic epidermal necrolysis. N Engl J Med 333: 1600-1607, 1995.

3. Paul C, Wolkenstein P, Adle H, Wechsler J, Garchon HJ, Revuz J and Roujeau JC: Apoptosis as a mechanism of keratinocyte death in toxic epidermal necrolysis. Br J Dermatol 134: 710-714, 1996.

4. Baron JM and Merk HF: Drug metabolism in the skin. Curr Opin Allergy Clin Immunol 1: 287-291, 2001. 
5. Wolkenstein $\mathrm{P}$, Tan $\mathrm{C}$, Lecoeur $\mathrm{S}$, et al: Covalent binding of carbamazepine reactive metabolites to $\mathrm{P} 450$ isoforms present in the skin. Chem Biol Interact 113: 39-50, 1998.

6. Rzany B, Hering O, Mockenhaupt M, Schröder W, Goerttler E, Ring J and Schöpf E: Histopathological and epidemiological characteristics of patients with erythema exudativum multiforme major, Stevens-Johnson syndrome and toxic epidermal necrolysis. Br J Dermatol 135: 6-11, 1996.

7. Paquet P, Nikkels A, Arrese JE, Vanderkelen A and Piérard GE: Macrophages and tumor necrosis factor-alpha in toxic epidermal necrolysis. Arch Dermatol 130: 605-608, 1994.

8. Viard I, Wehrli P, Bullani R, et al: Inhibition of toxic epidermal necrolysis by blockade of CD95 with human intravenous immunoglobulin. Science 282: 490-493, 1998.

9. Paquet P and Piérard GE: Epidermal calprotectin in druginduced toxic epidermal necrolysis. J Cutan Pathol 26: 301-305, 1999.

10. Paquet P, Jacob E, Pirson J and Piérard GE: Drug-induced toxic epidermal necrolysis and pancytopenia: A puzzling association. Int J Mol Med 16: 29-33, 2005.

11. Hetts S: To die or not to die: An overview of apoptosis and its role in disease. JAMA 279: 300-307, 1998.

12. Hung RWY and Chow AW: Dissecting the 'end game': clinical relevance, molecular mechanisms and laboratory assessment of apoptosis. Clin Invest Med 27: 324-344, 2004.

13. Morishima N, Nakanishi K, Takenacchi H, Shibata $T$ and Yasuhiko Y: An endoplasmic reticulum stress-specific caspase cascade in apoptosis. J Biol Chem 277: 34287-34294, 2002.

14. Rao RV, Castro-Obregon S, Frankowski H, et al: Coupling endoplasmic reticulum stress to the cell death program. J Biol Chem 277: 21836-21842, 2002.

15. Green DR and Reed JC: Mitochondria and apoptosis. Science 281: 1309-1312, 1998

16. Reed JC: Mechanisms of apoptosis avoidance in cancer. Curr Opin Oncol 11: 68-75, 1999.

17. Shear N, Spielberg S, Grant D, Tang BK and Kalow W: Differences in metabolism of sulfonamides predisposing to idiosyncratic toxicity. Ann Int Med 105: 179-184, 1986.

18. Cribb AG, Miller M, Leeder JS, Hill J and Spielberg SP: Reaction of the nitroso and hydroxylamine metabolites of sulfamethoxazole with reduced glutathione. Drug Metab Dispos 19: 900-906, 1991.

19. Cribb AE, Nakamura H, Grant DM, Miller MA and Spielberg SP: Role of the polymorphic and monomorphic human arylamine $\mathrm{N}$ acetyltransferases in determining sulfamethoxazole metabolism. Biochem Pharmacol 45: 1277-1282, 1993.

20. Grant DM, Blum M, Beer M and Meyer UA: Monomorphic and polymorphic human arylamine $\mathrm{N}$-acetyltransferases: a comparison of liver isozymes and expressed products of two cloned genes. Mol Pharmacol 39: 184-191, 1991.

21. Reilly T, Lash LH, Doll MA, Hein DW, Woster PM and Svensson CK: A role for bioactivation and covalent binding within epidermal keratinocytes in sulfonamide-induced cutaneous drug reactions. J Invest Dermatol 114: 1164-1173, 2000.

22. Dietrich A, Kawakubo Y, Rzany B, Mockenhaupt M, Simon JC and Schöpf E: Low N-acetylating capacity in patients with Stevens-Johnson syndrome and toxic epidermal necrolysis. Exp Dermatol 4: 313-316, 1995.

23. Herzenberg LA, Rosa SCD and Dubs JE: Glutathione deficiency is associated with compound survival in HIV diseases. Proc Natl Acad Sci USA 94: 1967-1972, 1997.

24. Staal FJT, Ela SW, Rodenrer M, Anderson MT and Herzenberg LA: Glutathione deficiency and human immunodeficiency virus infection. Lancet 339: 909-912, 1992.

25. Saiag P, Caumes E, Chosidow O, Revuz J and Roujeau JC: Drug-induced toxic epidermal necrolysis (Lyell syndrome) in patients infected with the human immunodeficiency virus. J Am Acad Dermatol 26: 567-574, 1992.
26. Hess D, Sisson M, Suria H, Wijsman J, Puvanesasungham R, Madrenas J and Rieders MJ: Cytotoxicity of sulfonamide reactive metabolites: apoptosis and selective toxicity of CD8+ cells by the hydroxylamine of sulfamethoxazole. FASEB J 13: 1688-1698, 1999.

27. Naisbitt DJ, Williams DP, Pirmohamed M, Kitteringham NR and Park BK: Reactive metabolites and their role in drug reactions. Curr Opin Allergy Clin Immunol 1: 317-325, 2001.

28. Shapiron LE and Shear NH: Mechanisms of drug reactions: the metabolic track. Semin Cutan Med Surg 15: 217-227, 1996.

29. Schreck R and Bauerle P: A role for oxygen radicals as second messengers. Trends Cell Biol 1: 39-42, 1991.

30. Schreck R, Albermann KAJ and Baeuerle P: Nuclear factor $\kappa ß$ : an oxidative stress-responsive transcription factor of eukaryotic cells (a review). Free Radic Res Comm 17: 221-237, 1992

31. Safarian T and Bredesen D: Is apoptosis mediated by reactive oxygen species? Free Radic Res 21: 1-8, 1994.

32. Lange RW, Hayden PJ, Chignell CF and Luster MI: Anthralin stimulates keratinocyte-derived proinflammatory cytokines via generation of reactive oxygen species. Inflamm Res 47: 174-181, 1998

33. Paquet $\mathrm{P}$ and Piérard GE: Soluble fractions of tumor necrosis factor- $\alpha$, interleukin 6 and of their receptors in toxic epidermal necrolysis: A comparison with second-degree burns. Int J Mol Med 1: 459-462, 1998.

34. Correia O, Delgado L, Leal Barbosa I, Campilho F and Fleming-Torrinha J: Increased interleukin 10, tumor necrosis factor $\alpha$, and interleukin 6 level in blister fluid of toxic epidermal necrolysis. J Am Acad Dermatol 47: 58-62, 2002.

35. Moncada S, Palmer RJ and Higgs EA: Nitric oxide: physiology, pathophysiology and pharmacology. Pharmacol Rev 43: 109-116, 1991.

36. Lerner LH, Qureshi AA, Reddy BV and Lerner EA: Nitric oxide synthase in toxic epidermal necrolysis and StevensJohnson syndrome. J Invest Dermatol 114: 196-199, 2000.

37. Ettore A, Andreassi M, Anselmi C, Neri P, Andreassi L and Di Stefano A: Involvement of oxidative stress in apoptosis induced by a mixture of isothiazolinones in normal human keratinocytes. J Invest Dermatol 121: 328-336, 2003.

38. Abe R, Shimizu T, Shibaki A, Nakamura H, Watanabe H and Shimizu H: Toxic epidermal necrolysis and Stevens-Johnson syndrome are induced by soluble Fas ligand. Am J Pathol 162: 1515-1520, 2003.

39. Arnold R, Seifert M, Asadullah K and Volk HD: Crosstalk between keratinocytes and T lymphocytes via Fas/Fas ligand interaction: modulation by cytokines. J Immunol 162: 7140-7147, 1999.

40. Halliwell B: Free radicals and antioxidants: a personal view. Nutr Rev 52: 253-265, 1994.

41. Bruch-Gerharz D, Ruzicka T and Kolb-Bachofen V: Nitric oxide in human skin: current status and future prospects. J Invest Dermatol 110: 1-7, 1998.

42. Kröncke KD, Fehsel K and Kolb-Bachofen V: Nitric oxide: cytotoxicity versus cytoprotection - how, why, when, and where? Nitric Oxide Biol Chem 1: 107-120, 1997.

43. Melino G and Bernassola F: S-nitrosylation regulates apoptosis. Nature 388: 432-433, 1997

44. Garcia-Doval I, Le Cleach L, Bocquet H, Otero XL and Roujeau JC: Toxic epidermal necrolysis and Stevens-Johnson syndrome: Does early withdrawal of causative drugs decrease the risk of death? Arch Dermatol 136: 323-327, 2000.

45. Redondo P, De Felipe I, De la Pena A, Azamendra JM and Vanaclocha U: Drug-induced hypersensitivity syndrome and toxic epidermal necrolysis treatment with $\mathrm{N}$-acetylcysteine. $\mathrm{Br} \mathrm{J}$ Dermatol 136: 633-634, 1997.

46. Velez A and Moreno JC: Toxic epidermal necrolysis treated with N-acetylcysteine. J Am Acad Dermatol 46: 469-470, 2002.

47. Paquet P, Piérard GE and Quatresooz P: Novel treatments for drug-induced toxic epidermal necrolysis (Lyell's syndrome). Int Arch Allergy Immunol 136: 205-216, 2005. 